\title{
Editorial
}

\section{Sensor Based Intelligent Systems for Detection and Diagnosis}

\author{
Mehmet Karakose \\ Computer Engineering, Firat University, 23119 Elazig, Turkey \\ Correspondence should be addressed to Mehmet Karakose; mkarakose@firat.edu.tr
}

Received 11 August 2015; Accepted 16 September 2015

Copyright (c) 2016 Mehmet Karakose. This is an open access article distributed under the Creative Commons Attribution License, which permits unrestricted use, distribution, and reproduction in any medium, provided the original work is properly cited.

Sensors are very commonly used as monitoring and observing elements in real world applications. However, sensors cannot provide a solution for systems, because data obtained with sensors should be processed for object functions. There are many approaches for processing and decisionmaking methods in industrial applications. In particular, it is very important for intelligent systems which used condition monitoring, detection, diagnosis, prognosis, and predictive maintenance.

The predictive maintenance basically consists of four components. These components are the condition monitoring, the detection, the diagnosis, and the prognosis for identification of anomalies and faulty conditions. The condition monitoring is monitoring of one or several parameters to identify an unexpected situation. The detection is indicating if there is an abnormal status using observable effects in a monitored system. The diagnosis is the process of detecting and determining where that anomaly is. The prognosis is predicting and estimating useful and healthy status of a system.

A lot of predictive maintenance techniques have been improved for detection, diagnosis, and prognosis of faults in the literature. The detection, diagnosis, and prognosis methods use measurements and preprocessed data using the condition monitoring. Then, the predictive maintenance methods provide a result for health of system. But systems have many uncertainties and they affect the performance of the predictive maintenance method. Therefore, intelligent systems are used to avoid this disadvantage. Process flow of the predictive maintenance is as follows. Firstly, actual functions or signals in any system for the condition monitoring are measured. Secondly, normal operation or faulty operation of the system for early recognition is determined in the anomaly detection. Thirdly, anomaly classification for correct assignment can be carried out for the diagnosis. Next, the prognosis stage can be used to estimate or predict anomalies. Finally, a result is obtained with decision-making.

An efficient predictive maintenance method should have some specifications. First of all, minimum information and sensor should be required. Other specifications are accurate and confident prediction, fast response, and robustness. Optimization algorithms such as genetic algorithms, artificial immune systems, and advanced Monte Carlo methods, learning algorithms such as artificial neural networks and support vector machines, and reasoning algorithms such as fuzzy logic systems, clustering algorithms, particle filtering algorithms, wavelet analysis algorithms, and principal component analysis algorithm can be used for the predictive maintenance methods. These methods can be used in data preprocessing, feature extraction, and feature selection too.

Finally, the predictive maintenance methods using computational intelligence techniques are very important for improving production and manufacturing and product quality. If the faulty condition in a system can be determined with the detection, the diagnosis, or the prognosis in early stage, most problems can be repaired at this time.

Mehmet Karakose 


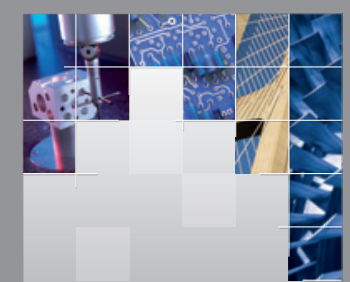

\section{Enfincering}
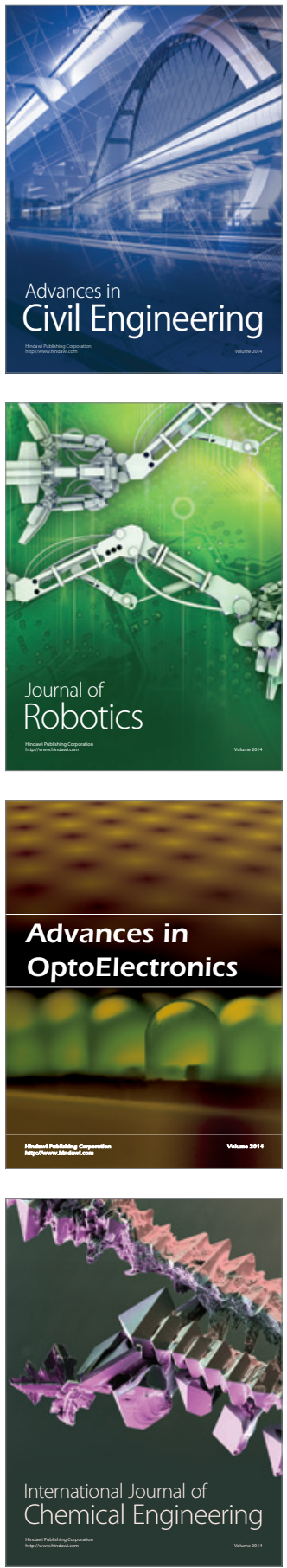

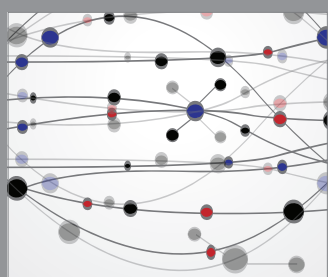

The Scientific World Journal

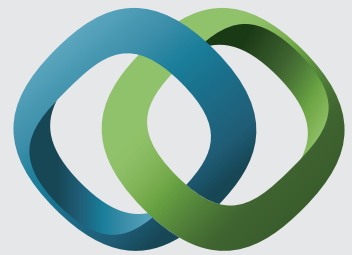

\section{Hindawi}

Submit your manuscripts at

http://www.hindawi.com
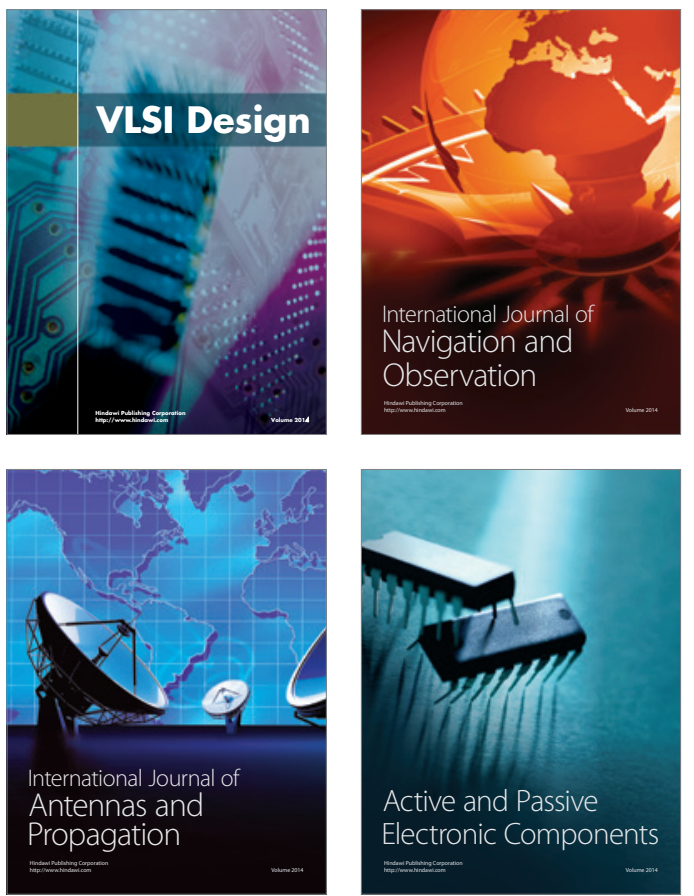
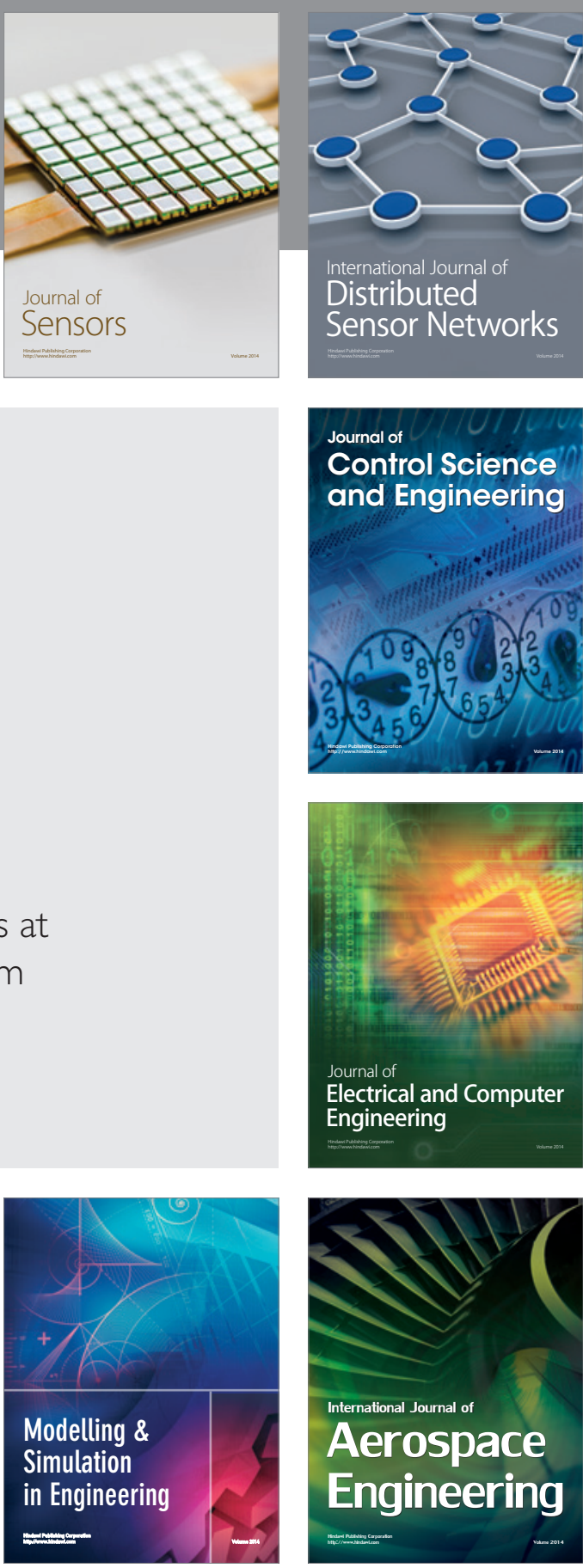

International Journal of

Distributed

Sensor Networks

Journal of

Control Science

and Engineering
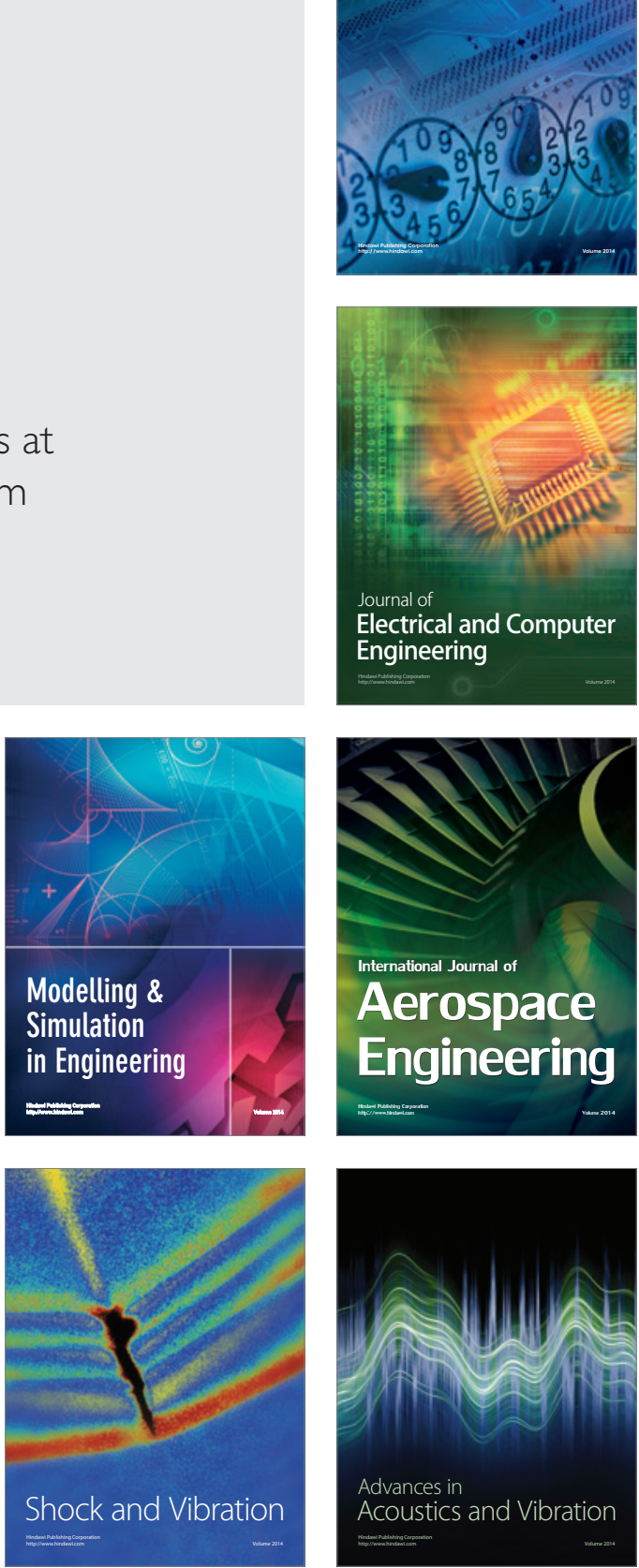